\title{
Treatment of midshaft clavicular delayed and non-unions with anteroinferior locking compression plating
}

\author{
Sjoerd A. Stufkens $\cdot$ P. Kloen
}

Received: 22 December 2008 / Published online: 2 April 2009

(C) The Author(s) 2009. This article is published with open access at Springerlink.com

\begin{abstract}
Introduction Pain and impaired shoulder function are the predominant symptoms of midshaft clavicle non-unions. Obtaining consolidation and improvement of shoulder function is often successfully achieved with osteosynthesis and bone grafting. Most data in the literature pertain to plate osteosynthesis, placing the plate on the subcutaneous superior aspect of the clavicle. Although union rates are generally high, most patients require hardware removal as the plate is prominent under the skin causing pain and cosmetic problems.

Materials and methods In the current retrospective study, we followed a cohort of 21 consecutive cases (20 patients) with a midshaft clavicular delayed or non-union, treated with anteroinferior plating using a $3.5 \mathrm{~mm}$ locking compression plate (LCP) for a mean of 30 months.

Results We operated on 10 males and 10 females with a mean age of 48.2 years (range 16-65). There was one early plate failure that needed revision. Two patients required hardware removal because of prominence of the plate. All but two patients were satisfied with the final cosmetic result. The average DASH score at follow up was 22.8.

Discussion and conclusions Anteroinferior plating with a $3.5 \mathrm{~mm} \mathrm{LCP}$ is a reliable and reproducible treatment of midshaft clavicular delayed and non-union regarding consolidation, function, cosmesis and reduction of second surgery.
\end{abstract}

S. A. Stufkens $(\bowtie) \cdot$ P. Kloen

Department of Orthopaedic Surgery, Academic Medical Center, PO Box 22660, 1100 DD Amsterdam, The Netherlands

e-mail: s.a.stufkens@amc.uva.nl

P. Kloen

e-mail: p.kloen@amc.uva.nl
Keywords Clavicula · Non-union · Delayed union · LCP . Locking compression plating $\cdot$ Anteroinferior placement

\section{Introduction}

Fractures of the clavicle comprise up to $15 \%$ of all adult upper extremity fractures. Most involve the midshaft and heal without operative treatment. Non-union rates have historically been reported to range from 0.1 to $4.8 \%$ after non-surgical management. This rate increases to $6.7 \%$ after acute surgical intervention [1-4]. A recent meta-analysis of 2,144 clavicle fractures contained 1,145 fractures treated conservatively, which resulted in a non-union rate of $5.9 \%$ [5]. Pain and decreased shoulder mobility are frequent invalidating complaints in a clavicular delayed and nonunion. Numerous reports have described their operative treatment. Fixation with plate and screws (with or without bone graft) is still considered the gold standard [6]. Hardware prominence often necessitates hardware removal after healing when the plate is positioned on the superior aspect of the clavicle. To prevent prominence of the plate, positioning the plate on the anteroinferior aspect of the clavicle is gaining popularity [7-9].

During the last 6 years, we have used the $3.5 \mathrm{~mm}$ pelvic locking compression plate (LCP) for anteroinferior plating of delayed unions and non-unions of the clavicula. This theoretically combines the following advantages: longer screw lengths provide a firmer construction at the maximal crosssectional length of the clavicle when approaching from anterior-inferiorly; low profile positioning obviates the need for hardware removal; angular stable screw fixation increases holding power in the lateral fragment; and maybe there is a lower susceptibility for axial pullout of the locking screws [10]. The purpose of this retrospective study is to describe 
patient characteristics and outcome of a consecutive series of 21 clavicular delayed and non-unions treated with anteroinferior plating using $3.5 \mathrm{~mm}$ pelvic LCP plates.

\section{Materials and methods}

\section{Patients}

Twenty patients (21 clavicles) were treated surgically for a midshaft delayed or non-union at the Academic Medical Center in Amsterdam from 2003 to 2007 by a single surgeon P. Kloen. We defined non-union as the lack of both periosteal and endosteal healing response, bridging the fracture after 6 months time. Delayed union was defined as lack of bridging callus between 3 and 6 months. In cases of doubt, a CT scan was performed to support or reject the diagnosis based on conventional radiographic images and clinical signs such as pain and weakness. Ten patients were female and ten were male. The mean age was 48.2 years (range 16-65). Initial fractures resulted all from trauma: 15 of them were falls in and around the house and during sports activities. Six non-unions were the result of a traffic accident, of which three motorcycle-related. The average duration of non-union before intervention in our clinic was 23 months (range 3-240). Clavicle fractures occurred in 13 $(62 \%)$ cases on the right side, in all but one case the dominant limb. Eighteen non-unions were atrophic with minimal shortening, three were hypertrophic. Pain was present in all 20 patients. Subjective weakness of the affected limb was only seen in five, all others thought they had not lost any arm strength. We did not use a force measuring device. One patient presented with a chronic wound dehiscence, one with complaints of hampered swallowing, which we were not completely able to understand, and one with paraesthesias. There were no pathologic fractures although one patient had radiotherapy including the clavicular area for Hodgkin lymphoma in the distant past. Fourteen patients were not operated previously. Six patients were initially operated on elsewhere: four directly after trauma, one after 2 months because of lacking callus and persisting disabling pain. One patient underwent multiple operations elsewhere before she presented to us with an infected non-union. In her case, the treatment with anteroinferior LCP was preceded by debridement, antibiotics and temporary stabilization using a $3.5 \mathrm{~mm}$ standard LCP as an external fixation device for 4 weeks to allow the open wound to heal. All other patients were operated by us only once.

\section{Surgical technique}

Under general anaesthesia, the patient was placed in a beach-chair position and draped allowing access to the clavicle and the ipsilateral iliac crest. No antibiotics were given until deep cultures were obtained. The incision was made just below the clavicle. In cases where a plate revision was performed, the incision was made over the scar and the skin pulled down to reach the anteroinferior side of the clavicle. A dissection was carried out carefully avoiding any injury to the subclavian vessels and the anterior fibres of the brachial plexus. When superior fibres of the pectoral muscle are abundant they can be cut and inserted under the plate later on. Locating the normal bone medial and lateral with regard to the non-union was generally easier allowing a 'safe' approach. After debridement of intervening tissue in the non-union with a knife or rongeur, both medullary canals were opened with a $2.0 \mathrm{~mm}$ drill. This was facilitated by holding the ends with a pointed reduction clamp. A $3.5 \mathrm{~mm}$ LCP was contoured to fit the serpentine shape of the anteroinferior clavicle optimally. The plate is first attached at the lateral side with at least one screw. In osteopenic bone, it might be prudent to use locking screws. The non-union was then reduced by pushing the lateral clavicle inward and upward. A small Verbrugge or bone reduction forceps can be applied around the plate and medial aspect. If possible a $3.5 \mathrm{~mm}$ cortical lag screw can be placed below the plate through the obliquity of the non-union. Fixation was then completed with additional screws medially and laterally. In most of our patients $(67 \%)$ we used a 7-hole plate. Harvesting cancellous bone from the iliac crest was done using a standard technique. Alternatively a commercially available bone graft substitute could be used. Decortication was done with a small osteotome at both non-union ends before the bone graft was placed. Postoperatively, the patients were given a sling for 10 days to allow wound healing. Pendulum and Codman exercises were started directly on the postoperative day. Active assisted range of motion exercises up to $90^{\circ}$ abduction and anteflexion were allowed in the first 6 weeks. Active unlimited motion exercises and strengthening were allowed when there was radiographic evidence of consolidation. Clinical and radiological assessment was performed generally at 6 weeks, 3 months, 6 months and 1 year (Fig. 1). For the purpose of this study, patients were invited to fill out a questionnaire at their latest follow-up or by mail: the authorized Dutch version of the DASH (Disabilities of the Arm, Shoulder and Hand). This score ranges from 0 to 100 points, where 0 points represent the best result. The mean follow-up time was 30 months (range 10-52 months).

\section{Results}

All 21 delayed and non-unions healed. For the daily activities/symptoms, the mean DASH score of our group was 22.8 (range 1.7-64.2), see Table 1. When the sports/arts 


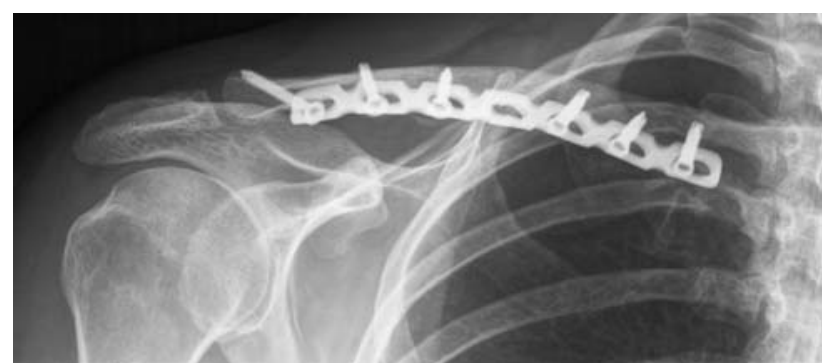

Fig. 1 Postoperative AP-radiograph of a previous midshaft clavicle non-union. There is consolidation achieved with a 7-hole $3.5 \mathrm{~mm} \mathrm{LCP}$ placed anteroinferiorly

and work sections were included the mean score was 23.6 (range 1.3-67.1). Clinical and radiographic union occurred in all delayed- and non-unions after a mean period of less than 3.5 months (range 2-6 months). Regarding the subjective outcome, all but three patients reported to be satisfied with the cosmetic result (Fig. 2a, b). Objective cosmetic outcome showed no prominence of the plates except in the three patients who were unhappy with the aspect of their shoulder. In two of these patients, the medial corner of the plate was prominent due to suboptimal contouring and therefore represents a technical error. One plate was removed, which rendered the patient free of complaints, the other is currently considering surgery. The third patient initially presented to us with a chronic infection and a large open wound showing the plate and multiple metal wires. A weight reduction of $20 \mathrm{~kg}$ after a successful two-stage procedure resulted in prominence of both the scar tissue and the hardware. Eventually, she requested removal of the metal and a scar revision that resulted in a satisfactory result. In 18 of the 20 cases, preoperative symptoms were eliminated after treatment; 18 out of $21(86 \%)$ plates placed anteroinferiorly required no final removal.

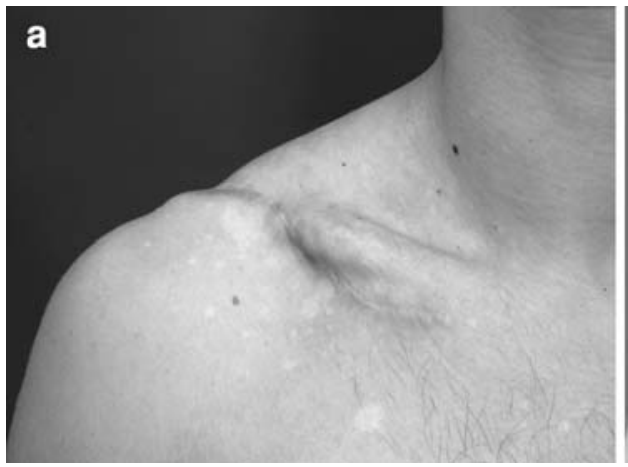

Fig. 2 a Postoperative conventional photograph of a patient operated on elsewhere, the plate being placed superiorly. Although the direction of the light and slenderness of the patient exaggerate the effect, still the plate will be always visible under the skin. The patient has had the plate

\section{Discussion and conclusion}

We found a mean DASH score of 22.8 at the latest followup, which indicates mild residual impairment. This is comparable to the outcome of anteroinferior plating with LCP of other authors [11]. Two patients had relatively bad scores at the last follow-up of 64.2 and 58.3 (Table 1). The first specifically noted that his chronic back pain was of influence on the DASH questions concerning daily activities. The second showed good shoulder and arm function on clinical follow-up. We were not able to identify the reason for his unfavourable overall functioning. One postoperative complication occurred in an obese (polytrauma) patient with type I diabetes mellitus. After delayed fixation of the clavicular fracture she suffered a plate failure 5 weeks after the surgery. We hypothesized a combination of excess weight and crutch ambulating because of her other injuries to be the cause of early plate failure. After reosteosynthesis with a similar plate the delayed union healed uneventfully. The patient remained unsatisfied, however, and refused to fill out a DASH score or attend a final follow-up to evaluate the function and appearance of her shoulder.

Regarding the cosmetical aspect, we have found no reports in the literature mentioning the benefit of anteroinferior placement of the plate instead of placing it directly underneath the skin. In our population, $85 \%$ of the patients were satisfied with the lack of protrusion of the plate. We consider the patient satisfaction with their appearance to be an important aspect of shoulder surgery. Moreover, less hindrance and irritation of the hardware makes second surgery abundant, thus optimal plate placement is of socioeconomical benefit as well.

We previously described twenty clavicle non- and malunions treated with a standard $3.5 \mathrm{~mm}$ reconstruction plate placed anteroinferiorly [9]. All healed within 2-8 months

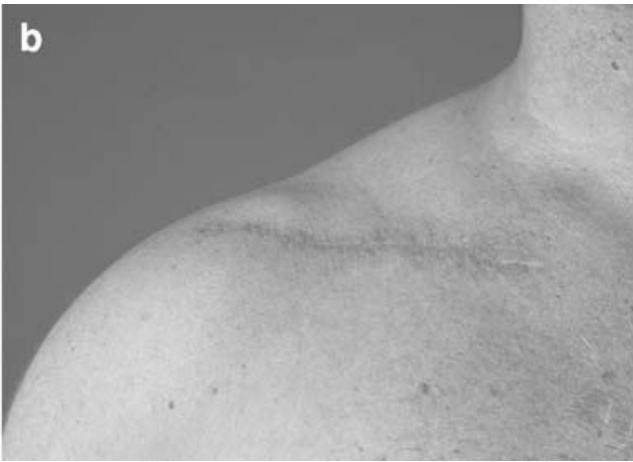

removed. b Postoperative conventional photograph of a patient from our series with the plate placed anteroinferiorly. Note that the plate is not visible under the skin. The patient is very satisfied with the cosmetic result 


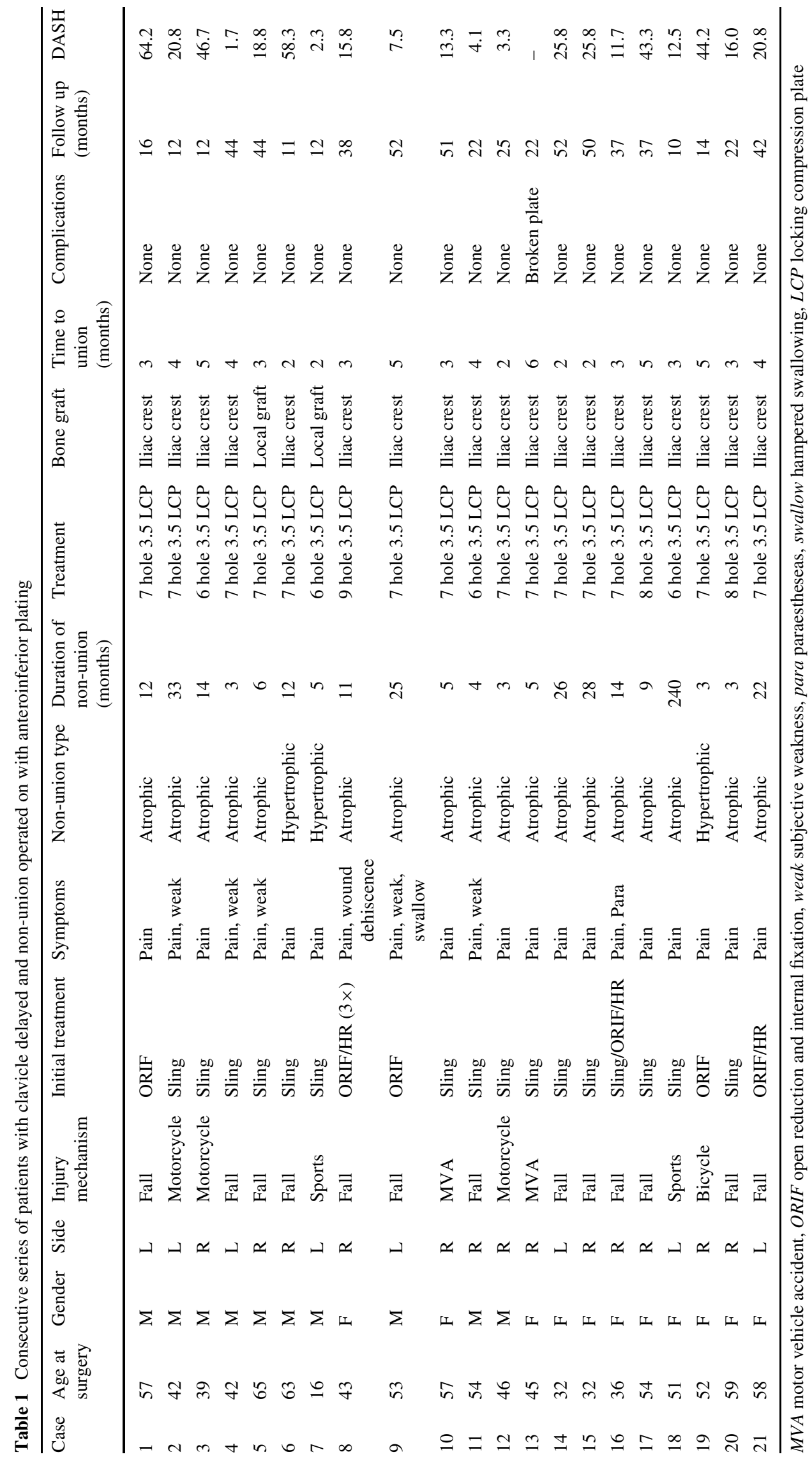


without significant complications. It is our experience that fewer patients request hardware removal when the plate is placed anteroinferiorly as it is less obtrusive [6]. Those patients who requested hardware removal had either a lag screw that was placed from superior to inferior outside of the plate or a suboptimal contouring of the plate which we regard an avoidable technical error.

Most surgeons place the plate on the superior surface of the clavicle because of the presumed tension band principle and the greater biomechanical stability shown in cadaver studies $[6,12,13]$. Coupe et al. [8] encountered a high incidence of complications via a traditional (superior) approach. Most of their 19 plates were removed, 15 because of prominence. Prominent and painful hardware is one of the main reasons for repeated intervention after consolidation $[14,15]$. Hardware removal is reported to be as high as $81.3 \%[1,16,17]$. As the indication for plate fixation of the fractured clavicle is increasing, based on recent level I evidence, decreasing the need for plate removal (thus requiring two surgeries) will both reduce the risk of harming the patient and will be of great financial benefit [18].

Limitations and weaknesses of this study consist of the lack of baseline function data, the small number of patients in our series and the lack of a control group. Unfortunately, we have failed to acquire DASH and/or Constant scores preoperatively because of the retrospective nature of the study. Perhaps obtaining preoperative data would have made a preoperative improvement of overall function visible. Another weakness of this study is the relatively small number of patients treated. Although the results appear good, percentages and significance of the DASH score are of limited value in small studies. Although non-union of mid-shaft clavicular fractures are more abundant than previously thought, the number of patients seen at our clinic remains low $[3,18,19]$. The third major limitation is the lack of a control group, for example, treated with the superior plating technique, with a different type of plate or with intramedullary nailing. We can therefore not state that our method is superior to different treatment methods. Biomechanical studies have shown that superior plating has mechanical advantages over anteroinferior plating because of the tension band principle [12]. We prefer the anteroinferior placement because the drilling is aimed away from the underlying neurovascular structures, the longer screw length that can be used providing more hold and less soft tissue irritation. We acknowledge that the technique is more difficult than superior plating.

We conclude that open reduction and internal fixation with a $3.5 \mathrm{~mm}$ pelvic LCP on the anteroinferior side of the clavicle with autologous bone grafting leads reproducibly to good results in delayed and non-unions. Moreover, a less prominent placing of the plate makes removal of the osteosynthesis material redundant.
Acknowledgment The authors did not receive any outside funding or grants in support of their research for or preparation of this work. Neither they nor a member of their immediate families received payments or other benefits or a commitment or agreement to provide such benefits from a commercial entity. No commercial entity paid or directed, or agreed to pay or direct, any benefits to any research fund, foundation, division, centre, clinical practice, or other charitable or non-profit organization with which the authors, or a member of their immediate families, are affiliated or associated.

Open Access This article is distributed under the terms of the Creative Commons Attribution Noncommercial License which permits any noncommercial use, distribution, and reproduction in any medium, provided the original author(s) and source are credited.

\section{References}

1. Eskola A, Vainionpaa S, Myllynen P, Patiala H, Rokkanen $P$ (1986) Outcome of clavicular fracture in 89 patients. Arch Orthop Trauma Surg 105(6):337-338

2. Olsen BS, Vaesel MT, Sojbjerg JO (1995) Treatment of midshaft clavicular nonunion with plate fixation and autologous bone grafting. J Shoulder Elbow Surg 4(5):337-344

3. Robinson CM (1998) Fractures of the clavicle in the adult. Epidemiology and classification. J Bone Joint Surg Br 80(3):476-484

4. Wilkins RM, Johnston RM (1983) Ununited fractures of the clavicle. J Bone Joint Surg Am 65(6):773-778

5. Zlowodzki M, Zelle BA, Cole PA, Jeray K, McKee MD (2005) Treatment of acute midshaft clavicle fractures: systematic review of 2144 fractures: on behalf of the Evidence-Based Orthopaedic Trauma Working Group. J Orthop Trauma 19(7):504-507

6. Jupiter JB, Leffert RD (1987) Non-union of the clavicle. Associated complications and surgical management. J Bone Joint Surg Am 69(5):753-760

7. Collinge C, Devinney S, Herscovici D, DiPasquale T, Sanders R (2006) Anterior-inferior plate fixation of middle-third fractures and nonunions of the clavicle. J Orthop Trauma 20(10):680-686

8. Coupe BD, Wimhurst JA, Indar R, Calder DA, Patel AD (2005) A new approach for plate fixation of midshaft clavicular fractures. Injury 36(10):1166-1171

9. Kloen P, Sorkin AT, Rubel IF, Helfet DL (2002) Anteroinferior plating of midshaft clavicular nonunions. J Orthop Trauma 16(6):425-430

10. Brouwer KM, Wright TC, Ring DC (2009) Failure of superior locking clavicle plate by axial pull-out of the lateral screws: a report of four cases. J Shoulder Elbow Surg 18(1):e22-e25

11. Khan SA, Shamshery P, Gupta V, Trikha V, Varshney MK, Kumar A (2008) Locking compression plate in long standing clavicular nonunions with poor bone stock. J Trauma 64(2):439-441

12. Celestre P, Roberston C, Mahar A, Oka R, Meunier M, Schwartz A (2008) Biomechanical evaluation of clavicle fracture plating techniques: does a locking plate provide improved stability? J Orthop Trauma 22(4):241-247

13. Iannotti MR, Crosby LA, Stafford P, Grayson G, Goulet R (2002) Effects of plate location and selection on the stability of midshaft clavicle osteotomies: a biomechanical study. J Shoulder Elbow Surg 11(5):457-462

14. Der TJ, Davison JN, Dias JJ (2002) Clavicular fracture non-union surgical outcome and complications. Injury 33(2):135-143

15. Rosenberg N, Neumann L, Wallace AW (2007) Functional outcome of surgical treatment of symptomatic nonunion and malunion of midshaft clavicle fractures. J Shoulder Elbow Surg 16(5):510-513 
16. Boehme D, Curtis RJ Jr, DeHaan JT, Kay SP, Young DC, Rockwood CA Jr (1991) Non-union of fractures of the mid-shaft of the clavicle. Treatment with a modified Hagie intramedullary pin and autogenous bone-grafting. J Bone Joint Surg Am 73(8):1219-1226

17. Mullaji AB, Jupiter JB (1994) Low-contact dynamic compression plating of the clavicle. Injury 25(1):41-45
18. Canadian Orthopaedic Trauma Society (2007) Nonoperative treatment compared with plate fixation of displaced midshaft clavicular fractures. A multicenter, randomized clinical trial. J Bone Joint Surg Am 89(1):1-10

19. Hill JM, McGuire MH, Crosby LA (1997) Closed treatment of displaced middle-third fractures of the clavicle gives poor results. J Bone Joint Surg Br 79(4):537-539 